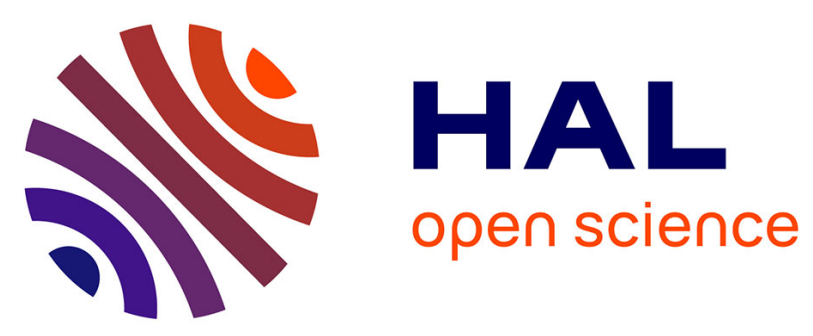

\title{
Premier signalement de la cochenille Maconellicoccus hirsutus (Green) en Guadeloupe; situation actuelle de ce ravageur des cultures dans les Antilles françaises (Hem., Pseudococcidae)
}

\author{
Danièle Matile-Ferrero, F. Leblanc, Daniel Marival
}

\section{To cite this version:}

Danièle Matile-Ferrero, F. Leblanc, Daniel Marival. Premier signalement de la cochenille Maconellicoccus hirsutus (Green) en Guadeloupe; situation actuelle de ce ravageur des cultures dans les Antilles françaises (Hem., Pseudococcidae). Bulletin de la Société Entomologique de France, 1998, 103 (2), pp.173-174. hal-02698126

\section{HAL Id: hal-02698126 \\ https://hal.inrae.fr/hal-02698126}

Submitted on 1 Jun 2020

HAL is a multi-disciplinary open access archive for the deposit and dissemination of scientific research documents, whether they are published or not. The documents may come from teaching and research institutions in France or abroad, or from public or private research centers.
L'archive ouverte pluridisciplinaire HAL, est destinée au dépôt et à la diffusion de documents scientifiques de niveau recherche, publiés ou non, émanant des établissements d'enseignement et de recherche français ou étrangers, des laboratoires publics ou privés. 
Jean ETIENNE, Danièle MATILE-FERRERO, Frédéric LEBLANC et Daniel MARIVAL:-

Premier signalement de Maconellicoccus hirsutus (Green) en Guadeloupe : situation actuelle de ce ravageur des cultures dans les Antilles françaises (Hemiptera, Pseudococcidae)

Summary - First record of the mealybug Maconellicoccus hirsutus (Green) from Guadeloupe ; present state of this pest of crops in the French Caribbean. Maconellicoccus hirsutus is recorded from Guadeloupe for the first time. The mealybug was accidentally introduced in the Caribbean recently (Grenada, Trinidad, St-Kitts, Nevis, St-Marteen and St-Barthelemy). Brief informations on biology, damage and control of the pest are given. The introduction of the entomophagous Coccinellid Cryptolaemus montrouzieri and the parasitoïd wasp Anagyrus kamali are recommended.

La cochenille Maconellicoccus hirsutus (Green) introduite accidentellement à Grenade en 1994, envahit progressivement l'arc caraïbe. Depuis, elle a en effet été signalée en 1995 à Trinidad (POLLARD, 1995 ; JONES, 1996) en 1996 à Saint-Kitts ( THOMAS \& EDWARDS, 1996) et à Nevis (WILLIAMS, 1996).

Aux Antilles françaises, $M$. hirsutus a été mentionné tout d'abord à Saint-Martin (MATILE-FERRERO \& ETIENNE, 1996) puis à Saint-Barthélémy (ETIENNE, 1997a). Nous signalons aujourd'hui sa présence en Guadeloupe où un premier foyer d'infestation vient d'être découvert le 16 avril 1998 à Capesterre Belle Eau, sur Allamanda cathartica, Alpinia purpurata, Artocarpus altilis, Citrus spp., Gliricidia sepium et Hibiscus rosa-sinensis.

Les dégâts provoqués par $M$. hirsutus sont caractéristiques des cochenilles farineuses, insectes suceurs de sève. Les cochenilles forment des colonies denses d'aspect cotonneux sur les plantes attaquées qui dépérissent en présentant des déformations spectaculaires (feuilles crispées) engendrées par la présence probable d'une toxine dans la salive de la cochenille (ETIENNE, 1997 b).

Cette espèce à cycle de développement rapide (3-4 semaines) à fécondité élevée (150 à 500 oeufs/femelle) (GHOSE, 1972) et à très grande polyphagie (plus de 125 plantes-hôtes recensées) représente un ravageur de toute première importance pour la Guadeloupe.

Dans l'immédiat, pour contenir les attaques de cette cochenille, des mesures prophylactiques comme la taille et le brulâge des parties attaquées, et dans certains cas, des mesures chimiques par utilisation de produits systémiques, sont à appliquer, tout en associant une lutte biologique complémentaire, indispensable pour un contrôle durable de ce nouveau ravageur. L'introduction des auxiliaires entomophages Cryptolaemus montrouzieri Mulsant (Coleoptera, Coccinellidae) et Anagyrus kamali Moursi (Hymenoptera, Encyrtidae) qui ont fait leur preuve, encore récemment à Saint-Martin dans le contrôle de cette cochenille, sont à recommander.

M. hirsutus menace désormais directement les Grandes Antilles (Porto Rico, République Dominicaine, Haiti, Jamaïque et Cuba) ainsi que les Etats-Unis (sud de la Floride) qui risquent, malgré les précautions prises, $\mathrm{d}^{\prime}$ être contaminés dans un avenir plus ou moins proche.

\section{AUTEURS CITES}

ETIENNE , J. , 1997a. - Mission à St-Barthélémy du 12 au 18 juillet 1997. Rapport de mission , 13p. 1997b. - Note sur la cochenille de l'Hibiscus et son contrôle biologique à Saint-Martin. Contrôle biologique à Saint-Martin, Fiche 1 : la cochenille de l'Hibiscus, INRA-FDGDEC Guadeloupe. 2p., avec 4 photos couleur GDEC Saint-Martin et INRA Valbonnne.

GHOSE , S.K., 1972. - Biology of the mealybug, Maconellicoccus hirsutus (Green) (Pseudococcidae, Hemiptera). Indian Agriculturist. 16 (4):323-332.

JONES, M.T., 1996. - Pink or Hibiscus Mealybug in Trinidad and Tobago.Caraphin News, 13:1-3.

MATILE-FERRERO, D. \& ETIENNE ,J., 1996. - Présence de la cochenille de l' Hibiscus,Maconellicoccus hirsutus à Saint-Martin (Hemiptera, Pseudococcidae). Revue française $d^{\prime}$ Entomologie., (N.S.) 18 (1):38.

POLLARD ,G.V., 1995. - Pink or Hibiscus Mealybug in the Caribbean.Caraphin News , 12: 1-2.

THOMAS ,J. \& EDWARDS , A.I., 1996. - The Pink Mealybug in St-Kitts .Caraphin News , $13: 3$

WILLLIAMS, D.J., 1996. - A brief account of the hibiscus mealybug Maconellicoccus hirsutus (Hemiptera : Pseudococcidae), a pest of agriculture and horticulture, with descriptions of two related species from southern Asia. Bulletin of entomological Research. 86:617-628.

(J.E. \& D.M. : Laboratoire d'Entomologie Lutte Intégrée, INRA, URPV, B.P. 515, 97165 Pointe-à-Pitre Cedex, Guadeloupe. - D. M-F. : Laboratoire d'Entomologie, Muséum national d'Histoire naturelle, 45, rue Buffon, F75005 Paris. - F. L. : CIRAD-FHLOR, Station de Neufchateau, 97130 Capesterre Belle-Eau, Guadeloupe). 